University of Wollongong

Research Online

Australian Institute for Innovative Materials -

Papers

Australian Institute for Innovative Materials

$1-1-2015$

Reduced graphene oxide and polypyrrole/reduced graphene oxide composite coated stretchable fabric electrodes for supercapacitor application

Chen Zhao

University of Wollongong, cz995@uowmail.edu.au

Kewei Shu

University of Wollongong, ks323@uowmail.edu.au

Caiyun Wang

University of Wollongong, caiyun@uow.edu.au

Sanjeev Gambhir

University of Wollongong, sanjeev@uow.edu.au

Gordon G. Wallace

University of Wollongong, gwallace@uow.edu.au

Follow this and additional works at: https://ro.uow.edu.au/aiimpapers

Part of the Engineering Commons, and the Physical Sciences and Mathematics Commons

Research Online is the open access institutional repository for the University of Wollongong. For further information contact the UOW Library: research-pubs@uow.edu.au 


\title{
Reduced graphene oxide and polypyrrole/reduced graphene oxide composite coated stretchable fabric electrodes for supercapacitor application
}

\author{
Abstract \\ The advent of self-powered functional garments has given rise to a demand for stretchable energy \\ storage devices that are amendable to integration into textile structures. The electromaterials (anode, \\ cathode and separator) are expected to sustain a deformation of $3 \%$ to $55 \%$ associated with body \\ movement. Here, we report a stretchable fabric supercapacitor electrode using commonly available nylon \\ lycra fabric as the substrate and graphene oxide (GO) as a dyestuff. It was prepared via a facile dyeing \\ approach followed by a mild chemical reduction. This reduced graphene oxide ( $\mathrm{rGO}$ ) coated fabric \\ electrode retains conductivity at an applied strain of up to $200 \%$. It delivers a specific capacitance of 12.3 \\ $\mathrm{F} \mathrm{g}^{-1}$ at a scan rate of $5 \mathrm{mV} \mathrm{s}^{-1}$ in $1.0 \mathrm{M}$ lithium sulfate aqueous solution. The capacitance is significantly \\ increased to $114 \mathrm{~F} \mathrm{~g}^{-1}$ with the addition of a chemically synthesized polypyrrole (PPy) coating. This PPy- \\ rGO-fabric electrode demonstrates an improved cycling stability and a higher capacitance at $50 \%$ strain \\ when compared to the performance observed with no strain.

\section{Keywords} \\ supercapacitor, electrodes, fabric, stretchable, graphene, coated, application, composite, reduced, \\ polypyrrole, oxide \\ Disciplines \\ Engineering | Physical Sciences and Mathematics

\section{Publication Details} \\ Zhao, C., Shu, K., Wang, C., Gambhir, S. \& Wallace, G. G. (2015). Reduced graphene oxide and polypyrrole/ \\ reduced graphene oxide composite coated stretchable fabric electrodes for supercapacitor application. \\ Electrochimica Acta, 172 12-19.
}


Reduced graphene oxide and polypyrrole/reduced graphene oxide composite coated stretchable fabric electrodes for supercapacitor

\section{application}

Chen Zhao, Kewei Shu, Caiyun Wang*, Sanjeev Gambhir, Gordon G. Wallace*

Intelligent Polymer Research Institute, ARC Centre of Excellence for Electromaterials

Science, University of Wollongong, NSW, 2522, Australia

\section{Corresponding authors:}

Caiyun Wang

Tel: +61 242981426

Fax: +61 242983114

E-mail: caiyun@uow.edu.au

Gordon G. Wallace:

Tel: +61 242213127

Fax: +61 242213124

E-mail: gwallace@uow.edu.au 


\section{Abstract}

The advent of self-powered functional garments has given rise to a demand for stretchable energy storage devices that are amendable to integration into textile structures. The electromaterials (anode, cathode and separator) are expected to sustain a deformation of $3 \%$ to $55 \%$ associated with body movement. Here, we report a stretchable fabric supercapacitor electrode using commonly available nylon lycra fabric as the substrate and graphene oxide (GO) as a dyestuff. It was prepared via a facile dyeing approach followed by a mild chemical reduction. This reduced graphene oxide (rGO) coated fabric electrode retains conductivity at an applied strain of up to 200\%. It delivers a specific capacitance of $12.3 \mathrm{~F} \mathrm{~g}^{-1}$ at a scan rate of $5 \mathrm{mV} \mathrm{s}^{-1}$ in 1.0 M lithium sulfate aqueous solution. The capacitance is significantly increased to 114 $\mathrm{F} \mathrm{g}^{-1}$ with the addition of a chemically synthesized polypyrrole (PPy) coating. This PPy-rGO-fabric electrode demonstrates an improved cycling stability and a higher capacitance at $50 \%$ strain when compared to the performance observed with no strain.

Keywords: stretchable electrode, graphene, polypyrrole, wearable, supercapacitor 


\section{Introduction}

Smart textiles, or E-textiles, are fabrics with embedded functional devices such as sensors, transistors or antennas [1-4]. This has gained tremendous interest in recent years and found widespread application in personalized electronics, wearable medical monitors, and even in the military field [5]. Textile based energy storage devices are needed to power such devices, since conventional batteries and supercapacitors are too bulky, rigid and heavy to be seamlessly integrated into wearable garments [6]. Compared to batteries, supercapacitors possess advantages in high power density, fast charging-discharging and extremely long cycling life [7]. Self-powered garments can be created by combining them with textile piezoelectrics [8] or thermoelectrics [9] to capture energy from human body movement or heat respectively.

These structures are expected to accommodate an average deformation of $3 \%$ to 55\% associated due to body movement [10]. There exists three main strategies to develop textile supercapacitors [11]: using coated textiles as electrodes [6, 12-20], fibres or yarns integrated into non-woven [21] or woven [22] textiles as electrodes, and fibre supercapacitors woven into textiles [23-28]. Among these approaches, coating the pre-existing textile structures with active electrode materials is the most facile and cost-effective technique [6].

Recently, diverse carbonaceous materials, such as single-walled carbon nanotubes (SWNTs) $[12,14]$, activated carbon $[6,16]$ and graphene $[13,15,20]$ have proven to be useful in fabricating coated textile electrodes. Conducting polymers, such as polypyrrole (PPy) [17-19], have also been used to produce textile based 
electrodes. A stretchable supercapacitor assembled with activated carbon coated textile electrodes exhibited a $\sim 30 \%$ capacitance loss with 50\% strain applied [16]. SWNTs coated textile electrodes could sustain a 120\% strain without obvious capacitance fade [12], and the chemically or electrochemically synthesized PPy/fabric electrodes even showed improved performance while being stretched [17, 18]. For large-scale applications, cost reduction of SWNTs is still required [13], and poor cycling stability of PPy should also be improved.

Graphene is a promising alternative to construct textile electrodes, as it can be produced from inexpensive graphite at a large-scale via cost-effective chemical methods. Recently, graphene based textile electrodes demonstrated high capacitance and flexibility $[13,15,20]$. Solution processed graphene coated textiles delivered a specific capacitance of $\sim 63 \mathrm{~F} \mathrm{~g}^{-1}$ [13]. A high specific capacitance of $326.8 \mathrm{~F} \mathrm{~g}^{-1}$ was achieved by a thermally annealed graphene coated cotton cloth [15]. Graphene coated electrospun polyamide-66 nanofabrics exhibited a specific capacitance of $280 \mathrm{~F} \mathrm{~g}^{-1}$ [20]. However, the stretchability of those textile electrodes was not discussed.

In this work, a stretchable conductive fabric electrode was prepared using GO as a dyestuff and nylon lycra fabric as the substrate. A facile dyeing approach followed by a mild chemical reduction was employed. An additional coating of redox-active and highly conducive PPy film will further enhance the performance of this fabric electrode. PPy can be integrated with graphene nanosheets through the $\pi-\pi$ stacking interaction between the rGO sheet and the conjugated backbones of PPy [29, 30]. Currently reported PPy/rGO composites are either in powder or sheet form, they can’t 
be stretched. Here, PPy was chemically polymerized onto the conductive rGO coated fabric to achieve a stretchable PPy/rGO/fabric composite electrode. rGO sheets play the role of stabilizer for PPy, improving the poor cycling stability of PPy as described in our previous work [17]. The electrochemical properties of these fabric electrodes under strain were investigated.

\section{Experimental}

\subsection{Reagents and materials}

Nylon lycra fabric was purchased from Spotlight Stores Pty Ltd. Pyrrole (Py) was sourced from Merck. Naphthalene-2,6-disulfonic acid disodium salt $\left(\mathrm{Na}_{2} \mathrm{NDS}\right)$, ammonium persulfate (APS) and lithium sulfate monohydrate $\left(\mathrm{Li}_{2} \mathrm{SO}_{4} \mathrm{H}_{2} \mathrm{O}\right)$ were obtained from Sigma-Aldrich. L-ascorbic acid (L-AA) was purchased from Alfa-Aesar. Pyrrole was freshly distilled, whereas other chemicals were used as supplied. All aqueous solutions were prepared using Milli-Q water ( 18 M $\Omega$ ).

\subsection{Preparation of rGO coated fabric}

Graphite oxide was synthesized from graphite flakes using the modified Hummers’ method [31, 32]. Graphite oxide was exfoliated to graphene oxide using a Branson Digital Sonifier (S450D, 30\% amplitude, $2 \mathrm{~s}$ on and $1 \mathrm{~s}$ off) for $30 \mathrm{~min}$, forming a stable suspension with a concentration of $3 \mathrm{mg} \mathrm{mL}^{-1}$. The nylon lycra fabric was treated with an aqueous solution of scouring agent and sodium hydroxide for $1 \mathrm{~h}$, followed by rinsing with abundant water. After drying, it was treated with plasma to increase the wettability prior to the dyeing process.

The fabric $\left(1 \times 3 \mathrm{~cm}^{2}\right)$ was immersed into a GO suspension for $30 \mathrm{~min}$ at room 
temperature and then dried at $60{ }^{\circ} \mathrm{C}$ for $30 \mathrm{~min}$. This process was repeated to increase the GO uptake. The reduction of GO coated fabric was performed by immersing into $60 \mathrm{~mL}$ of $0.1 \mathrm{M} \mathrm{L}$-ascorbic acid solution $\left(95^{\circ} \mathrm{C}\right.$ ) for $60 \mathrm{~min}$ with constant stirring. Such dyed fabric was washed with abundant water and finally dried at $60{ }^{\circ} \mathrm{C}$ under vacuum overnight. The resultant fabric is denoted as rGO-fabric.

\subsection{Preparation of PPy modified rGO-fabric}

PPy was chemically polymerized onto the rGO-fabric according to our previous report with minor modification [17]. Briefly, rGO-fabric $\left(1 \times 3 \mathrm{~cm}^{2}\right)$ was immersed in a $20 \mathrm{~mL}$ aqueous solution containing Py and $\mathrm{Na}_{2} \mathrm{NDS}$. $5 \mathrm{~mL}$ ethanol was added to improve the wettability of rGO-fabric. The solution was kept stirring in an ice bath for $30 \mathrm{~min}$, followed by addition of $5 \mathrm{~mL}$ pre-cooled APS solution drop-wise to initiate polymerization. The reaction was performed in an ice-bath for 0.5 to $3 \mathrm{~h}$. The concentration of Py, APS and $\mathrm{Na}_{2} \mathrm{NDS}$ was $0.02 \mathrm{M}, 0.02 \mathrm{M}$ and $0.009 \mathrm{M}$, respectively. The coated fabric was washed with abundant water, followed by sonication in water for 30 min to remove the loosely bound PPy. Finally, the fabric was dried at $40{ }^{\circ} \mathrm{C}$ under vacuum overnight. The obtained fabric composite is coded as PPy-rGO-fabric. The fabric electrodes with $2 \mathrm{~h}$ PPy deposition showed the highest specific capacitance. Only these samples were chosen for the investigation in this work. The loading amount of PPy was $\sim 2.8 \mathrm{mg} \mathrm{cm}^{-2}$.

\subsection{Characterization}

Physicochemical properties: XRD patterns were recorded by a powder X-ray diffraction system (GBC MMA, $\mathrm{Cu} \mathrm{K} \alpha$ radiation, $\lambda=0.15418 \mathrm{~nm}$ ). Raman spectra 
were obtained using a confocal Raman spectroscope (Jobin Yvon HR800, Horiba) utilizing $632.8 \mathrm{~nm}$ diode lasers. GO was drop-casted on the substrate (quartz for XRD and glass slide for Raman) and dried for the characterization. The morphology of the fabric electrodes was characterized by FE-SEM (JEOL JSM-7500FA). The surface resistivity of the fabric electrode was measured according to the American Association of Textile Chemists and Colourists Test Method 76-1995 [33]: two rectangular copper electrodes were placed on the sample and the surface resistivity $\left(\rho_{\mathrm{s}}\right)$ was given by:

$$
\rho_{\mathrm{s}}=\mathrm{R} \times \frac{\mathrm{L}}{\mathrm{D}}
$$

Where $\rho_{\mathrm{s}}$ is the surface resistivity $\left(\Omega \mathrm{sq}^{-1}\right)$, R is the surface resistance $(\Omega)$ measured by the multimeter, $\mathrm{L}$ is the length of the copper electrode and $\mathrm{D}$ is the separation distance between the copper electrodes.

Tensile-strain tests of the fabric electrode were conducted on a Shimadzu EZ mechanical tester with a $20 \mathrm{~mm}$ gauge length and a $5.5 \mathrm{~mm}$ width at a speed of $1.67 \%$ $\mathrm{s}^{-1}$. The in-situ resistance changes of the electrode were measured by an Agilent 34410A digital multimeter during the stretching process at a speed of $0.167 \% \mathrm{~s}^{-1}$. The cyclic stretching-releasing test was performed at a speed of $5 \% \mathrm{~s}^{-1}$.

Electrochemical properties: A symmetric supercapacitor device was assembled to test the electrochemical properties of the fabric electrodes. Two pieces of fabric electrodes $\left(1 \times 3 \mathrm{~cm}^{2}\right)$ attached to two pieces of nickel foam were separated with a filter paper to form a sandwich structure. 1.0 $\mathrm{M} \mathrm{Li}_{2} \mathrm{SO}_{4}$ aqueous solution was the electrolyte. 
Cyclic voltammetry (CV) was conducted over the range of 0 to $0.8 \mathrm{~V}$ using a CHI 650D electrochemical workstation (CHI instruments, USA). The specific capacitance of a single electrode was given by [34]:

$$
C_{s p}=\frac{2}{s m \Delta V} \int i d V
$$

Where $\mathrm{s}\left(\mathrm{V} \mathrm{s}^{-1}\right)$ is the scan rate, $\mathrm{m}(\mathrm{g})$ is the rGO loading on a single electrode, $\Delta \mathrm{V}(\mathrm{V})$ is the potential range in one sweep segment and $\mathrm{i}(\mathrm{A})$ is the voltammetric current. Electrochemical impedance spectra (EIS) were measured using a Gamry EIS 3000 system over the frequency range of $100 \mathrm{kHz}$ to $0.01 \mathrm{~Hz}$ with an AC perturbation of 10 $\mathrm{mV}$ at open circuit potential. Galvanostatic charge-discharge tests were performed using a battery test system (Neware electronic Co., China) between 0 and $0.8 \mathrm{~V}$.

\section{Results and Discussion}

XRD and Raman spectra were utilized to characterize the structure of GO used in this work. GO showed a strong and sharp diffraction peak at $10.8^{\circ}$ in its XRD pattern (Fig. 1a), corresponding to an interlayer spacing of $\sim 0.8 \mathrm{~nm}$. It is much larger than that of graphite $(\sim 0.34 \mathrm{~nm})$ due to the introduction of hydroxyl, epoxy, carboxyl and carbonyl groups on the basal planes and edges of GO sheets [35]. This degree of oxygenation enabled GO to be readily dispersed in water. Moreover, these functional groups can form hydrogen bonding with the amide group of nylon, allowing the attachment of GO sheets to the nylon fibres during the dyeing process [20].

In the Raman spectrum of graphite (Fig. 1b), the band at $1334 \mathrm{~cm}^{-1}$ (D band) was caused by the disorders in the graphite edges, and the $G$ band at $1583 \mathrm{~cm}^{-1}$ was attributed to the first order scattering of the $\mathrm{E}_{2 \mathrm{~g}}$ mode of hexagonal carbon lattice. For 
GO, the D band increased in intensity, which can be attributed to the reduced size of the in-plane $\mathrm{sp}^{2}$ domains caused by the oxidation [36]. The $\mathrm{G}$ band broadened and shifted to higher frequencies $\left(1595 \mathrm{~cm}^{-1}\right)$. The shift may be due to the presence of double bonds [37].

3.1 Properties of rGO coated stretchable conductive fabric

Buglione et al. have reported that the methods used to reduce GO have a dramatic influence upon the capacitance, and that thermally reduced GO exhibited the highest values [38]. However, thermal reduction is limited in this work because of the low stability of the fabric at high temperature. Chemical reduction can be realized at a relatively low temperature. L-AA can reduce GO under mild conditions and the oxidized products are eco-friendly [39, 40]. Clear color changes were observed in the digital images of the original fabric, fabric coated with GO and after reduction with L-AA (Fig. 2a-c). The color changed from dark brown to black after reduction, and this is associated with the restoration of the electronic conjugation. The textile treatment procedure described in the experimental section resulted in a uniform and adherent coating of GO. The raw fabric showed an intertwining structure (Fig. 2d) with bundles of smooth fibres (Fig. 2e). After dyeing and reduction, rGO coated not only on the surface of the fibres but also filled the interstitial spaces (Fig. $2 \mathrm{f}$ and $\mathrm{g}$ ). rGO displayed a wrinkled surface morphology (Fig. 2g).

Raman spectra were obtained to characterize the chemical structure transformations of the fabric composites, as shown in Fig. 3. The spectrum of the raw fabric displayed characteristic peaks assigned to nylon. The peak at $1440 \mathrm{~cm}^{-1}$ 
represented the $\mathrm{CH}_{2}$ bending region in the C-C backbone. Peaks at $1122 \mathrm{~cm}^{-1}, 1277$ $\mathrm{cm}^{-1}, 1306 \mathrm{~cm}^{-1}, 1600 \mathrm{~cm}^{-1}$ and $2898 \mathrm{~cm}^{-1}$ were assigned to the C-C stretching, amide III group, $\mathrm{CH}_{2}$ twisting, amide I group and the symmetric $\mathrm{C}-\mathrm{H}$ stretching, respectively [41]. The GO coated fabric exhibited two prominent broad peaks: D band at $1330 \mathrm{~cm}^{-1}$ and $\mathrm{G}$ band at $1590 \mathrm{~cm}^{-1}$, confirming successful coating of GO on the nylon fibres. The intensity ratio of $\mathrm{D}$ and $\mathrm{G}$ band $\left(\mathrm{I}_{\mathrm{D}} / \mathrm{I}_{\mathrm{G}}\right)$ increased after chemical reduction (from 1.31 to 1.60 ), which can be ascribed to the decrease in the average size of the $\mathrm{sp}^{2}$ domains associated with the reduction of GO. As new graphitic domains were created upon reduction, their sizes were smaller than the ones in GO, but the quantity increased [36]. The background peaks of the fabric were weakened after reduction treatment may be explained by that rGO sheets stacked more densely on the fibres after the removal of oxygenated functional groups.

The rGO-fabric was electrically conductive, and the level increased with the number of dyeing cycles. The repeated dyeing process resulted in an increased loading amount of rGO on the fabric (Fig. 4a). This can be ascribed to the improved orientation and rGO sheet-to-sheet connection on the fabric surface. After 25 dyeing cycles, a surface resistivity of $240 \Omega \mathrm{sq}^{-1}$ was obtained with an rGO loading of $2.3 \mathrm{mg}$ $\mathrm{cm}^{-2}$. The surface resistivity did not change noticeably after being subjected to further dyeing cycles, so the rGO-fabric with 25 dyeing cycles was chosen for the following tests.

The tensile tests results of the fabric composites are shown in Fig. 4b. The tensile test of rGO-fabric gave a force-strain plot which exhibited a higher slope compared 
with the uncoated fabric, which means a higher force was required for rGO-fabric to be stretched to the same strain. The elongation at break of rGO-fabric was lower than that of uncoated fabric. A higher force required and lower elongation at break can be ascribed to the decrease in the inter-fibre sliding and increased stiffness induced by the rGO coating [42].

The electrical resistance of rGO-fabric was evaluated with strain up to 200\% (Fig. 4c). The electrode retains conductivity even at 200\% elongation. At strains lower than $50 \%$, the resistance decreased with the increased strain. When the strain was beyond $50 \%$, the resistance increased as the strain increased. Generally, the fabric consisted of continuous yarns which were intertwined into loops. The individual fibres in those yarns can be abstracted into single helical coiled springs [43]. The strain applied to the fabric was accommodated by the shape change of the coiled structure rather than the deformation of rGO coating. No cracks can be found on the rGO coating when $50 \%$ strain was applied to the fabric (Fig S1). Those fibres inside the fabric were straightened firstly under strain, resulting in more efficient contact between the rGO coated fibres leading to an enhancement in conductivity. An increase in resistance occurred at larger strains due to the loss of percolation of the rGO sheets on the fibres.

A maximum strain of 50\% was applied for the cyclic elongation-relaxation test to avoid the irreversible deformation induced with large strains. The normalized resistance decreased when it was stretched, and increased during the relaxation process (Fig. 4d). It was found to be irreversibly increased after 1000 stretching cycles at a $50 \%$ strain, from $240 \Omega \mathrm{sq}^{-1}$ to $1000 \Omega \mathrm{sq}^{-1}$. The fabric electrode 
demonstrated a plastic deformation of $10 \%$.

\subsection{Electrochemical properties of rGO-fabric}

The electrochemical properties of the rGO-fabric were tested in $1.0 \mathrm{M} \mathrm{Li}_{2} \mathrm{SO}_{4}$ aqueous solution in a two-electrode configuration. $\mathrm{Li}_{2} \mathrm{SO}_{4}$ was chosen as the electrolyte since carbon materials can achieve higher capacitance in this electrolyte because of the slow $\mathrm{H}_{3} \mathrm{O}^{+}$ion sorption [44].

The supercapacitors composed of rGO-fabric without or with $50 \%$ strain all showed nearly rectangular CV curves even at a high scan rate of $100 \mathrm{mV} \mathrm{s}^{-1}$ (Fig. 5a and b), indicating fast ion diffusions in the electrodes. It delivered a specific capacitance of 12.3, 10.9, 9.5, 7.8 and $6.6 \mathrm{~F} \mathrm{~g}^{-1}$ at a scan rate of 5, 10, 20, 50 and 100 $\mathrm{mV} \mathrm{s}^{-1}$, respectively. At a $50 \%$ strain, the corresponding capacitances increased to 15.5, 14.3, 12.7, 10.4 and $9.2 \mathrm{~F} \mathrm{~g}^{-1}$, respectively (Fig. 5c). The rGO-fabric showed higher capacitance at 50\% strain, which is consistent with the PPy coated nylon-lycra fabric [17]. This improvement might be due to the easy accessibility of ions at the electrode-electrolyte interface and the increased electrical conductivity of rGO-fabric induced by the applied strain.

In EIS characterization, the supercapacitors with fabric electrodes at $0 \%$ and $50 \%$ strain all exhibited a compressed semicircle in the high frequency region and a straight line in the low frequency region in the Nyquist plots (Fig. 5d). At high frequency, the intercept point on the real axis represents the resistance of the electrolyte, the internal resistance of the electrodes and the contact resistance of the active materials and current collectors: equivalent series resistance (ESR). Our system 
exhibited a very small ESR of approximately $0.75 \Omega$ and $0.55 \Omega$ at $0 \%$ and $50 \%$ strain, respectively. The diameter of the compressed semicircle in the high frequency region affords the charge-transfer resistance in the system, which decreased with increasing strain. This result is consistent with the CV testing.

The charge-discharge profiles of the supercapacitors at a current density of $0.1 \mathrm{~A}$ $\mathrm{g}^{-1}$ are presented in Fig. 5e. The duration of one full charge-discharge cycle became longer with strain applied (from 39 s to 56 s), indicating an increased capacitance. This result was consistent with those obtained from CV tests. The rGO-fabric delivered a specific capacitance of $10 \mathrm{~F} \mathrm{~g}^{-1}$ at this current density, and it increased to $13.5 \mathrm{~F} \mathrm{~g}^{-1}$ with $50 \%$ strain applied. The cycling stabilities of the supercapacitors were assessed at $0.1 \mathrm{~A} \mathrm{~g}^{-1}$ for 2000 cycles. The rGO-fabric at 50\% strain also exhibited improved cycling stability (Fig. 5f). The capacitance retention was 89\%, compared to $76 \%$ at $0 \%$ strain. The calculated Coulombic efficiency was kept above $94 \%$ in the whole testing cycles.

It is also noticed that the specific capacitance of the rGO-fabric was not high. The low capacitance can be ascribed to the limited accessible surface area due to the restacking of rGO nanosheets. However, its high conductivity and stretchability allow it to be a promising stretchable conductive substrate for the deposition of pseudocapacitive materials, such as conducting polymers, to further improve its electrochemical properties. In this work, PPy was deposited on the stretchable rGO-fabric to improve its performance.

3.3 Properties of PPy-rGO-fabric 
It can be seen clearly that after chemical polymerization, PPy was formed on the surface of the rGO coated fibres (Fig. 6a). The PPy layer was composed of PPy nodules in different sizes (Fig. 6b). With 50\% strain applied, there were no cracks forming on the PPy coating (Fig. S2). The existence of PPy is also evidenced from the Raman spectrum (Fig. 6c). The peaks at 925, 990 and $1047 \mathrm{~cm}^{-1}$ were assigned to the C-H out of plane deformation, pyrrole ring deformation and $\mathrm{C}-\mathrm{H}$ in plane deformation, respectively. Two strong peaks at 1328 and $1565 \mathrm{~cm}^{-1}$ represented the ring stretching and C-C backbone stretching, respectively [45]. These two peaks were overlapped with the D band and G band of rGO.

Although it has been reported that the capacitance of PPy can be affected by the electrolyte [46], to keep the consistency with rGO-fabric and calculate the capacitance contribution of PPy properly, the same electrolyte, $1.0 \mathrm{M} \mathrm{Li}_{2} \mathrm{SO}_{4}$ aqueous solution, was employed for the PPy-rGO-fabric electrode. The CV curves of the supercapacitors composed of PPy-rGO-fabric without and with 50\% strain retained a rectangular shape at scan rates up to $50 \mathrm{mV} \mathrm{s}^{-1}$. When the scan rate reached $100 \mathrm{mV}$ $\mathrm{s}^{-1}$, the CV curves became distorted (Fig 7a and b). This can be attributed to the inclusion/ejection and diffusion of counter-ions being slow compared to the electron transfer in the PPy matrix at high scan rates $[47,48]$. The PPy-rGO-fabric delivered a specific capacitance of $114,103,96,78$, and $46 \mathrm{~F} \mathrm{~g}^{-1}$ without strain at a scan rate of 5 , 10, 20, 50, and $100 \mathrm{mV} \mathrm{s}^{-1}$, respectively (based on the total mass of rGO and PPy) (Fig 7c). The specific capacitance was remarkably enhanced due to the additional pseudocapacitance originating from PPy. At $5 \mathrm{mV} \mathrm{s}^{-1}$, the energy density of our 
assembled supercapacitor is $2.53 \mathrm{Wh} \mathrm{kg}^{-1}$. With $50 \%$ strain applied, the corresponding specific capacitance was slightly increased to $125,112,99,81$, and $47 \mathrm{~F} \mathrm{~g} \mathrm{~g}^{-1}$, respectively over the above scan rate range investigated. At a current density of $0.5 \mathrm{~A}$ $\mathrm{g}^{-1}$, it delivered a specific capacitance of $93 \mathrm{~F} \mathrm{~g}^{-1}$, and it increased slightly to $100 \mathrm{~F} \mathrm{~g}^{-1}$ with 50\% strain applied (Fig S3).

In order to evaluate the capacitive contribution from PPy, its specific capacitance was calculated. It was found that PPy delivered a specific capacitance of 198, 179, 167, 136 and $79 \mathrm{~F} \mathrm{~g}^{-1}$ (based on the amount of PPy) over the scan rates range investigated. This specific capacitance is higher than that delivered from PPy (123.3 F $\mathrm{g}^{-1}$ at $10 \mathrm{mV} \mathrm{s}^{-1}$ ) which was chemically polymerized directly on the nylon lycra fabric (PPy-fabric) [17]. Moreover, a capacitance loss of 56\% was observed for this fabric electrode as the scan rate increased from 10 to $100 \mathrm{mV} \mathrm{s}^{-1}$, which is lower than $68 \%$ for the PPy-fabric. The rGO sheets acted as inner current collectors and formed a conductive network under the PPy layer, facilitating electron transfer between PPy and rGO, resulting in more efficient utilization of PPy in this work.

The cycling stabilities of the supercapacitors were evaluated at $0.5 \mathrm{~A} \mathrm{~g}^{-1}$ for 1000 cycles. The PPy-rGO-fabric also showed an improved cycling stability with 50\% strain applied (Fig. 7d). 79\% of capacitance was preserved for the fabric electrode with $50 \%$ strain, compared to that of $74 \%$ without strain applied. This can be explained by the improved conductivity with strain as described in rGO-fabric part. The calculated Coulombic efficiency was kept above $90 \%$ in the whole testing cycles without or with strain applied. This capacitance retention was inferior to that of the 
rGO-fabric. This can be due to the mechanical degradation of the PPy layer during the doping-dedoping process. However, it demonstrated an improved cycling stability compared with PPy-fabric. A capacitance retention of 76\% was observed after 500 cycles, in sharp contrast to that of only $12.5 \%$ for PPy-fabric [17]. Here, PPy nanoparticles were tightly connected to the rGO sheets due to the $\pi$ - $\pi$ stacking interaction between the rGO sheet and the conjugated backbones of PPy [30]. The rGO sheets acted as frameworks to stabilize PPy, limiting swelling and shrinking of the PPy coating during cycling. The capacitance loss of PPy was minimized, resulting in an improved cycling stability.

\section{Conclusions}

In conclusion, we report a stretchable and conductive rGO coated fabric electrode prepared via a facile dyeing approach. The rGO coated fabric had a surface resistivity of $240 \Omega \mathrm{sq}^{-1}$ and could sustain its conductivity with strain up to $200 \%$. This conductive electrode exhibited a low specific capacitance of $12.3 \mathrm{~F} \mathrm{~g}^{-1}$ at a scan rate of $5 \mathrm{mV} \mathrm{s}^{-1}$ in $1.0 \mathrm{M} \mathrm{Li}_{2} \mathrm{SO}_{4}$. However, it can be used as a stretchable conductive substrate for pseudocapacitive materials deposition. The specific capacitance increased significantly to $114 \mathrm{~F} \mathrm{~g}^{-1}$ at $5 \mathrm{mV} \mathrm{s}^{-1}$ with a PPy coating on this rGO-fabric via chemical polymerization. The rGO sheets formed a conductive network under the PPy layer, facilitating electron transfer between PPy and rGO, leading to a high capacitance contribution of $198 \mathrm{~F} \mathrm{~g}^{-1}$ originating from PPy. Furthermore, PPy attached on the rGO sheet tightly through $\pi-\pi$ stacking interaction, swelling and shrinking caused by doping-dedoping was accommodated by the rGO sheets network, resulting 
in an improved cycling stability. These fabric electrodes showed improved performance under strain. The potential window of this cell can be enlarged by constructing the asymmetric cell coupled with carbon based electrodes [49].

\section{Acknowledgments}

The authors thank the Australian Research Council (ARC) for financial support under the ARC Centre of Excellence for Electromaterials Science. Chen Zhao and Kewei Shu acknowledge the support of their CSC scholarships from the Ministry of Education of P. R. China. The authors acknowledge the use of facilities within the UOW Electron Microscopy Centre, and ANFF Materials Node for their provision of research facilities. The authors also thank A/Prof. Chee O. Too for proof-reading of this manuscript. 

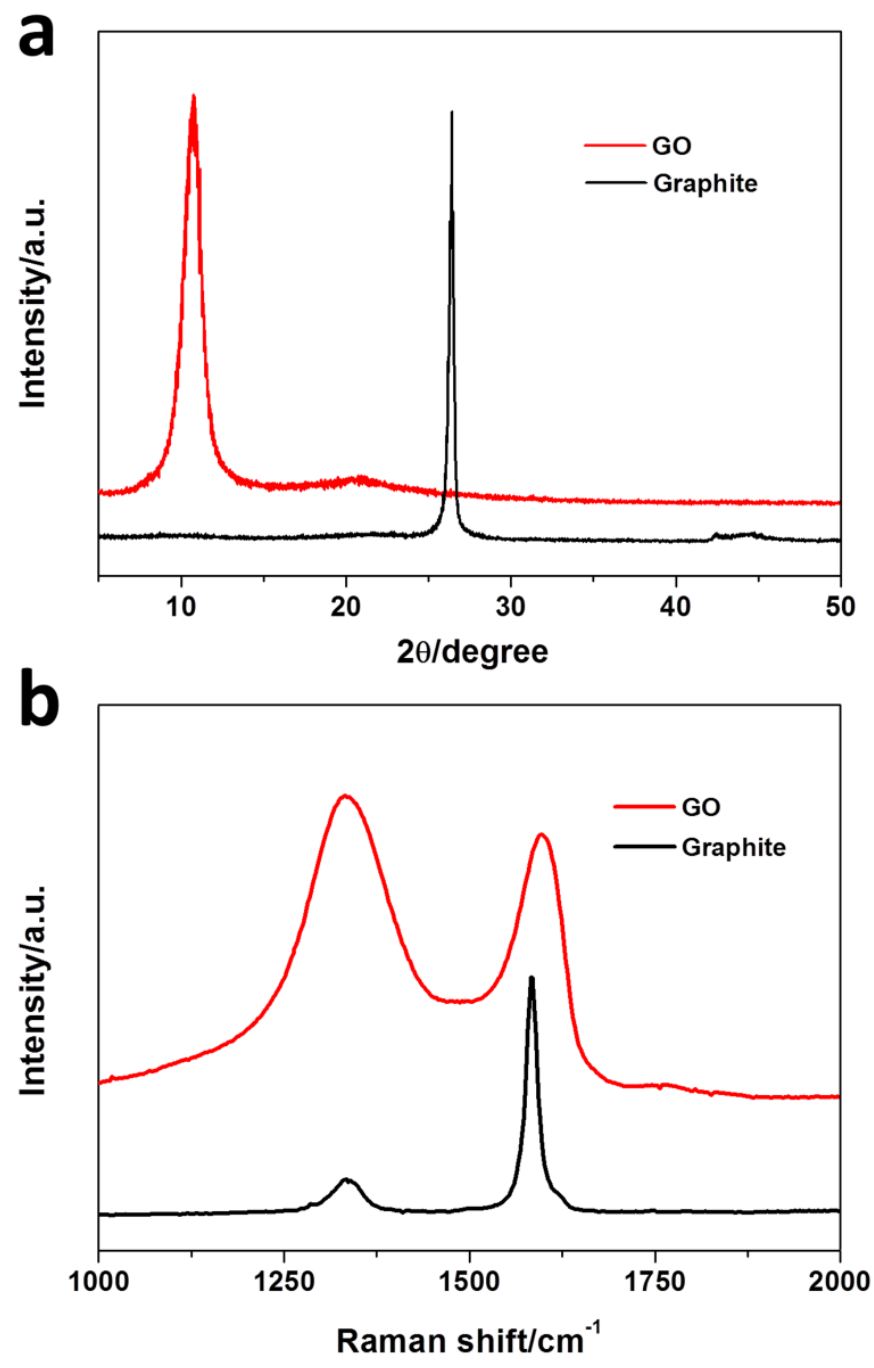

Fig. 1 XRD patterns (a) and Raman spectra (b) of GO and graphite 


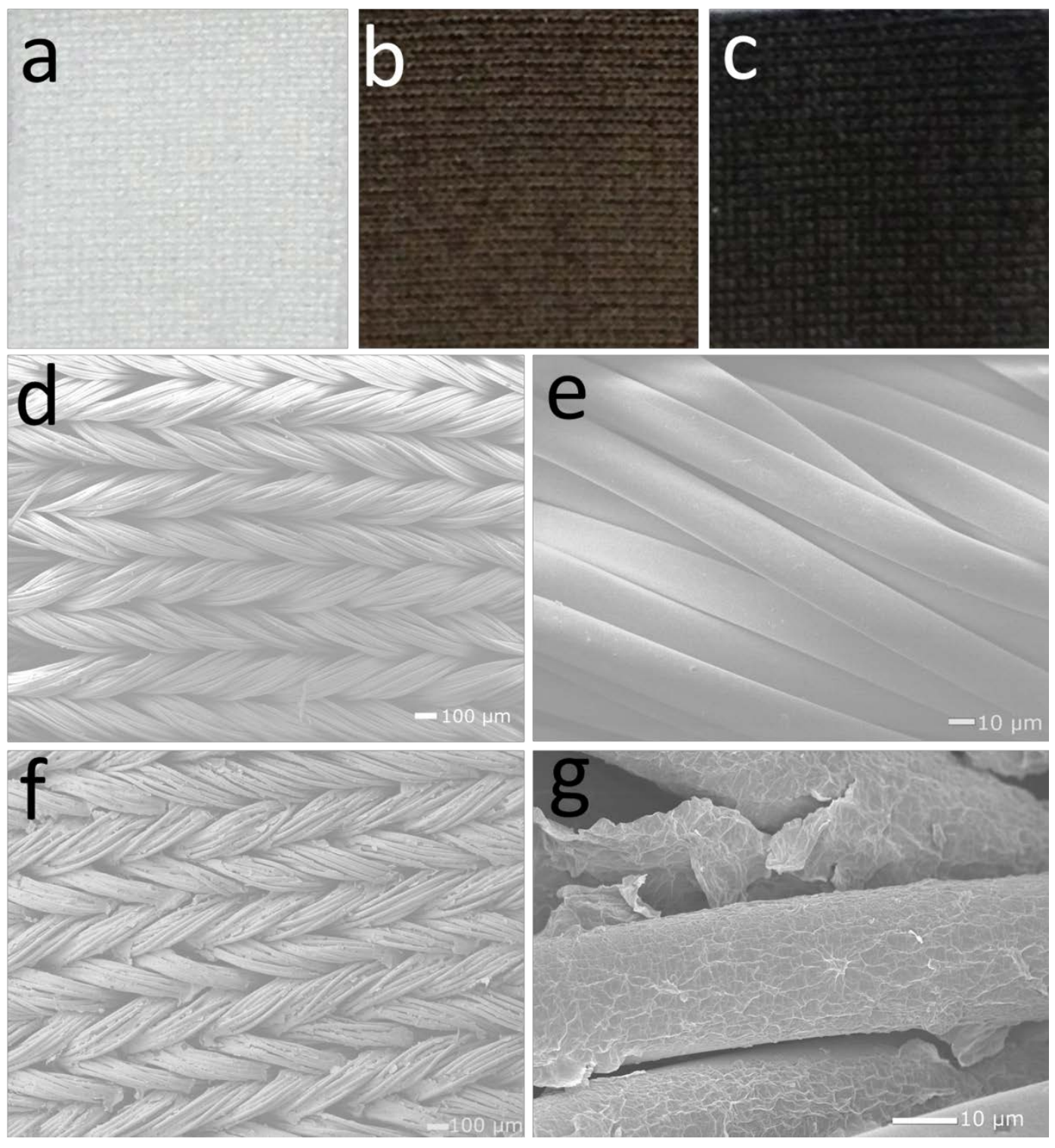

Fig. 2 Digital images of raw fabric (a), GO coated fabric (b) and rGO coated fabric (c).

SEM images of raw fabric (d, e) and rGO coated fabric (f, g). 


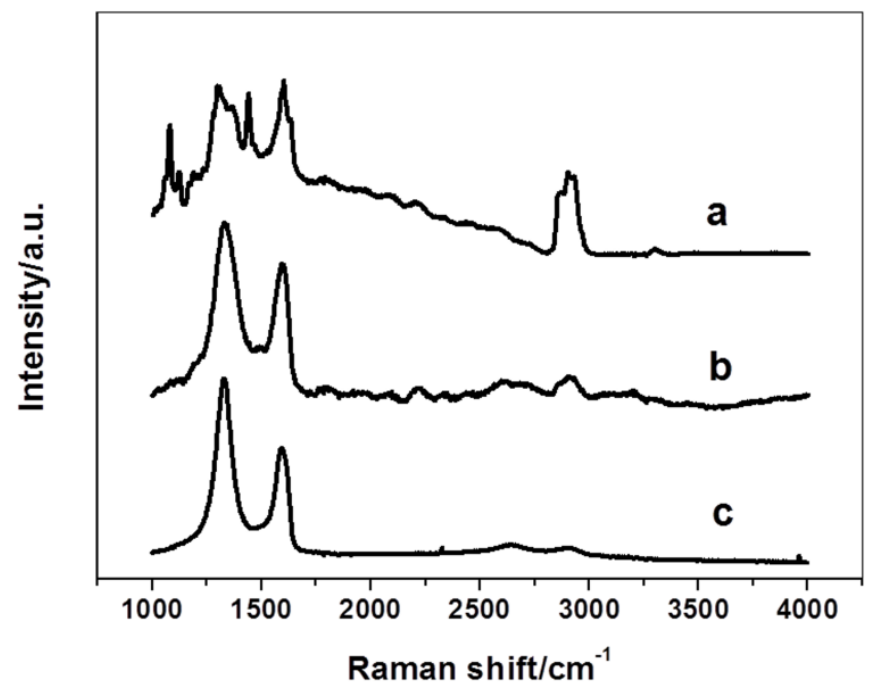

Fig. 3 Raman spectra of the raw fabric (a), GO coated fabric (b) and rGO coated fabric (c). 

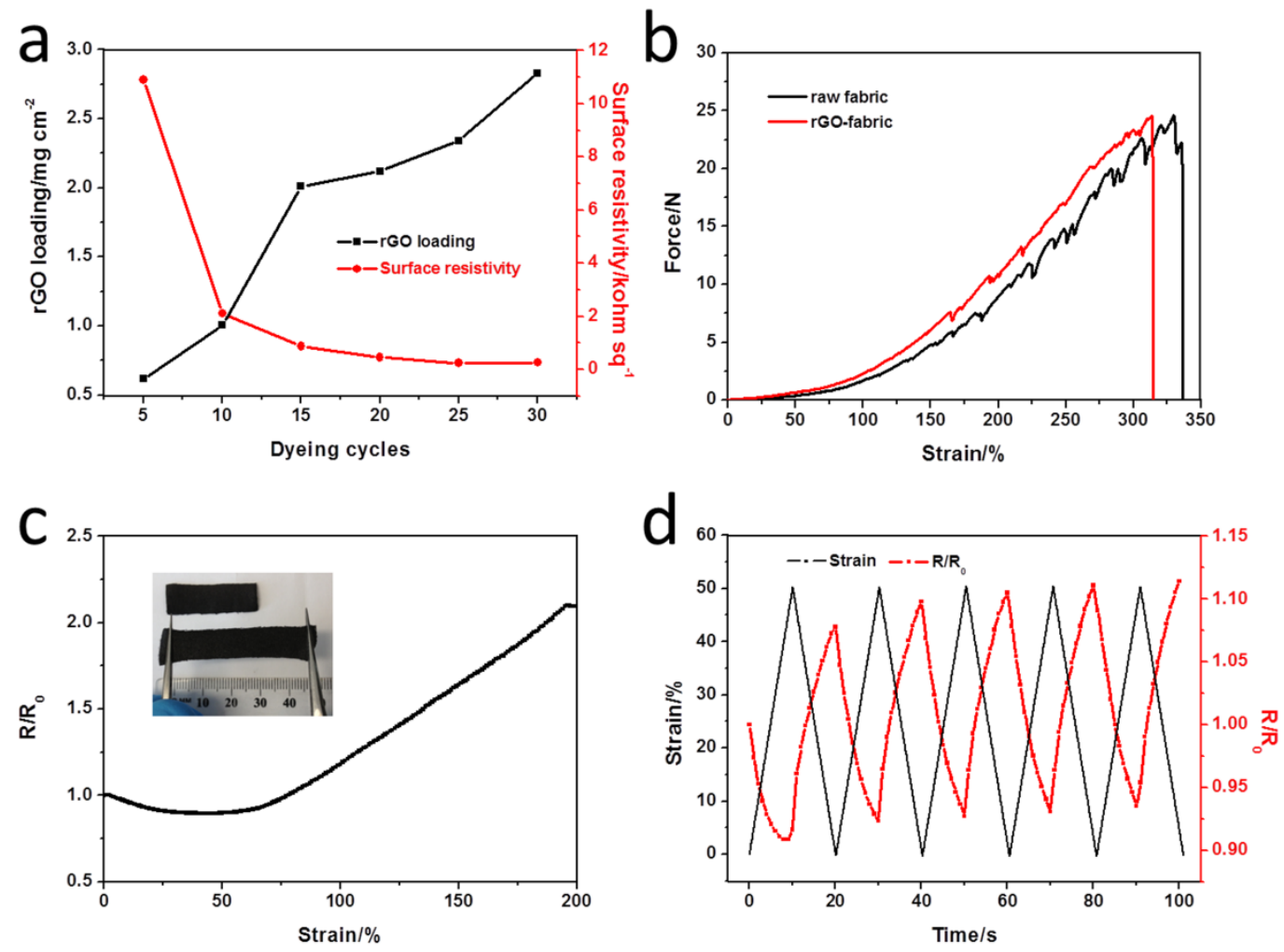

Fig. 4 (a) rGO loading and surface resistivity of the fabric as a function of dyeing cycles. (b) Tensile tests of raw fabric and rGO-fabric. (c) Normalized resistance versus strain of rGO-fabric (Inset, rGO-fabric at relaxation and a 50\% strain). (d) Normalized resistance changes of rGO-fabric under cyclic strain of $50 \%$ (first 5 cycles). 

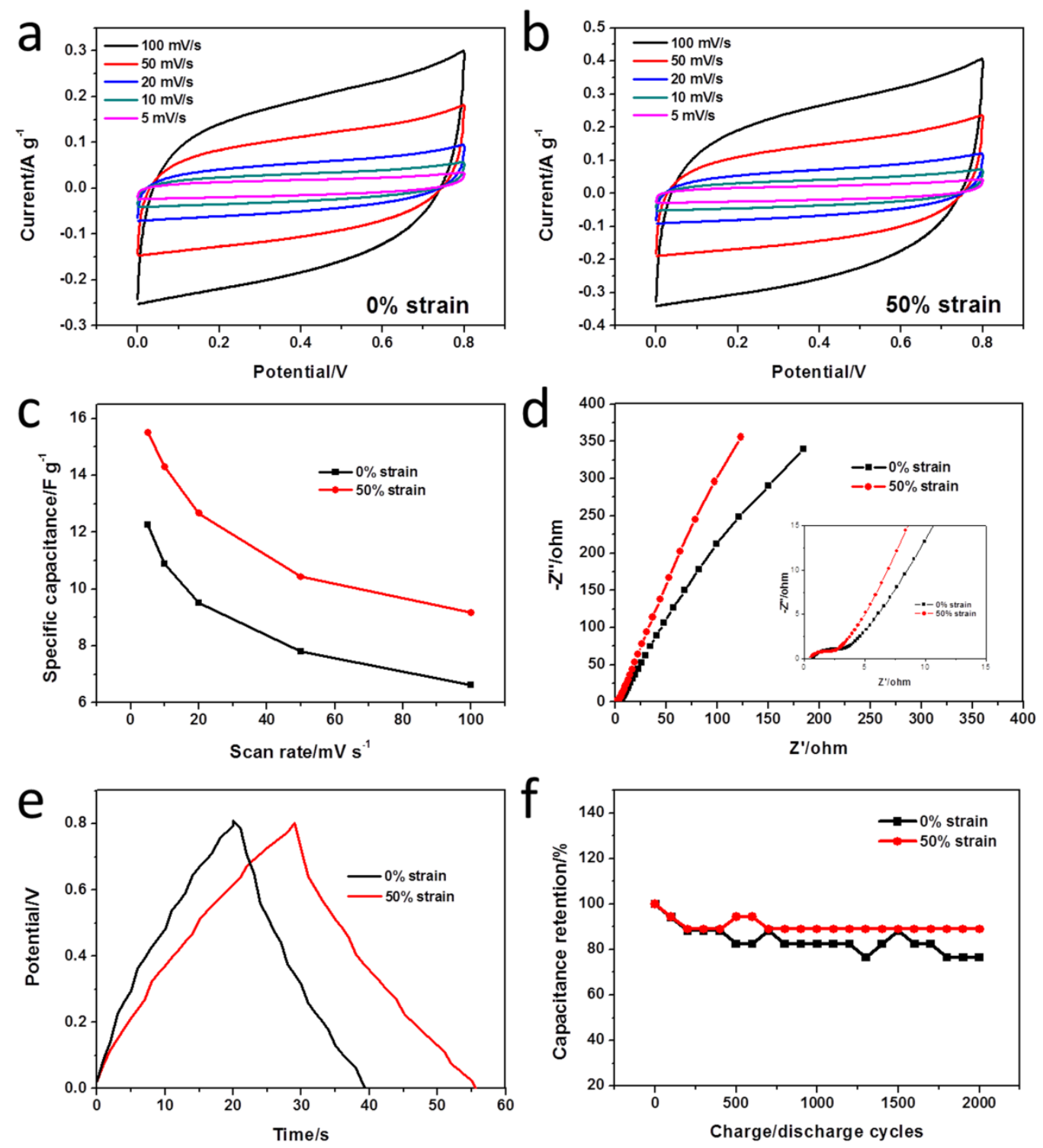

Fig. 5 CV curves of the supercapacitors composed of rGO-fabric at $0 \%$ (a) and $50 \%$

(b) strain. (c) Specific capacitances of the rGO-fabric at $0 \%$ and $50 \%$ strain. (d)

Nyquist plots. (e) Charge-discharge profiles, and (f) capacitance retention of supercapacitors composed of rGO-fabric at $0 \%$ and $50 \%$ strain. 

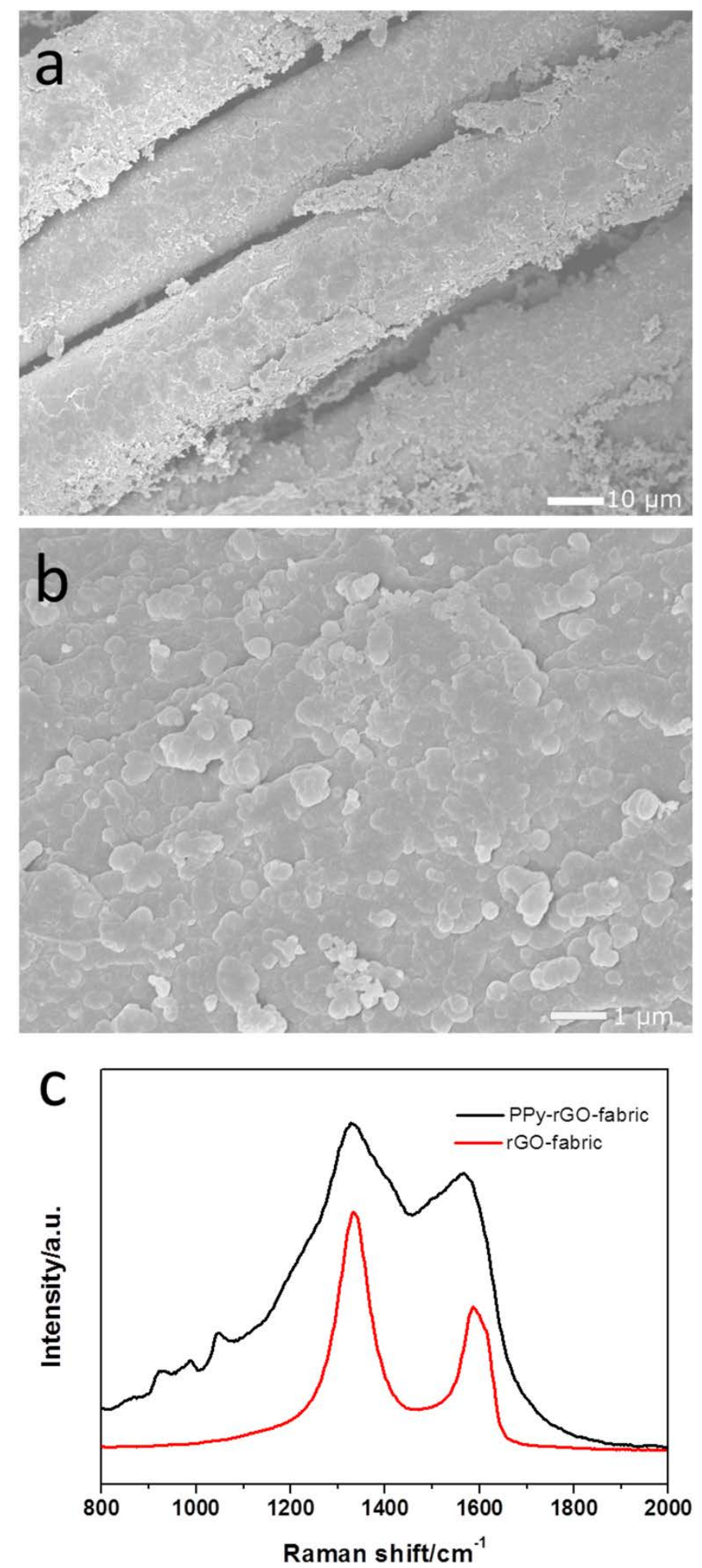

Fig. 6 SEM images of PPy-rGO-fabric at low (a) and high (b) magnification. (c) Raman spectrum of PPy-rGO-fabric and rGO-fabric. 

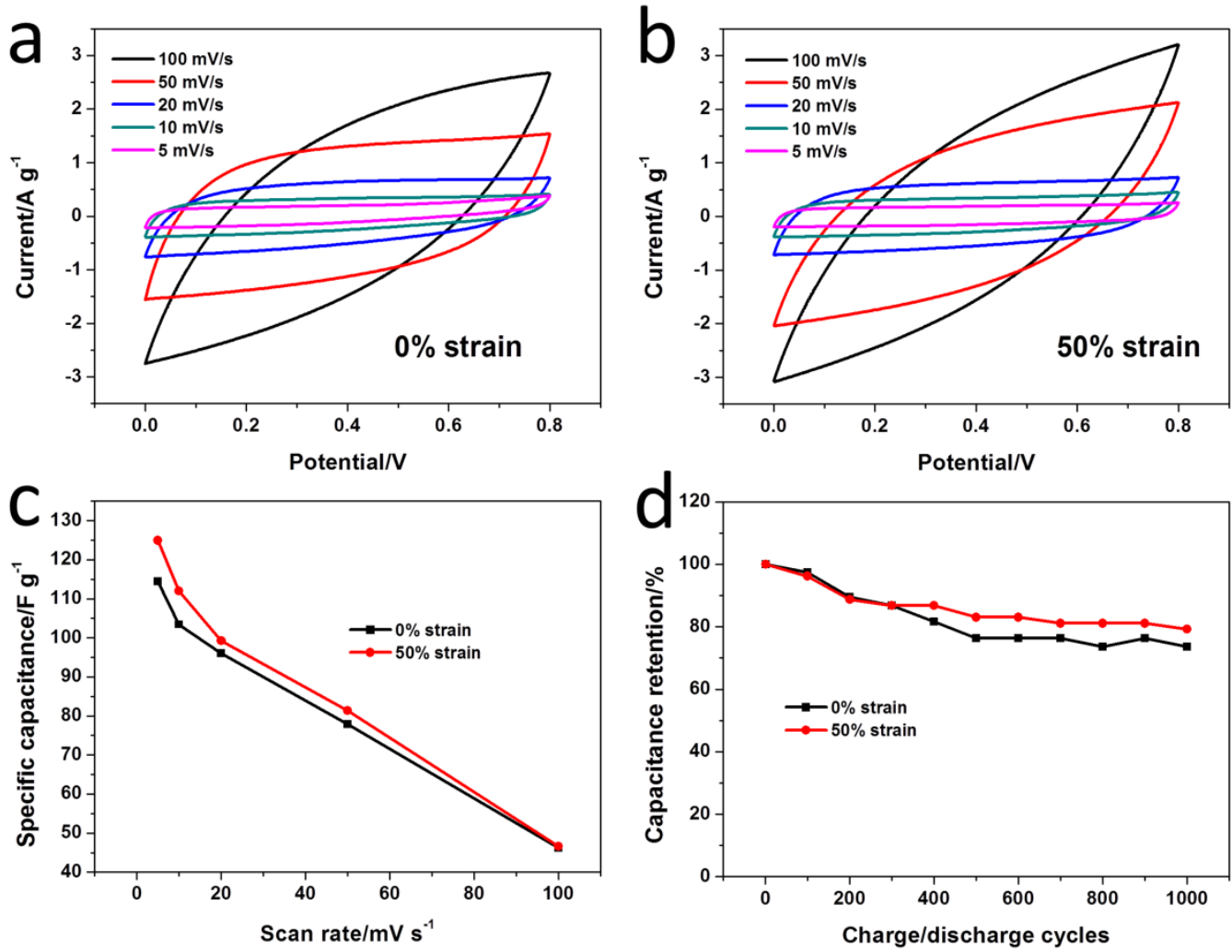

Fig. 7 CV curves of the supercapacitors composed of PPy-rGO-fabric at $0 \%$ (a) and 50\% (b) strain. (c) Specific capacitances of the PPy-rGO-fabric at 0\% and 50\% strain. (d) Capacitance retention of the supercapacitors composed of PPy-rGO-fabric at $0 \%$ and $50 \%$ strain. 


\section{References}

[1] T. Yamada, Y. Hayamizu, Y. Yamamoto, Y. Yomogida, A. Izadi-Najafabadi, D.N. Futaba, K. Hata, A stretchable carbon nanotube strain sensor for human-motion detection, Nat. Nanotechnol. 6 (2011) 296.

[2] M. Hamedi, R. Forchheimer, O. Inganäs, Towards woven logic from organic electronic fibres, Nat. Mater. 6 (2007) 357.

[3] C. Müller, M. Hamedi, R. Karlsson, R. Jansson, R. Marcilla, M. Hedhammar, O. Inganäs, Woven electrochemical transistors on silk fibers, Adv. Mater. 23 (2011) 898. [4] R. Salvado, C. Loss, R. Gonçalves, P. Pinho, Textile materials for the design of wearable antennas: a survey, Sensors 12 (2012) 15841.

[5] S. Coyle, Y. Wu, K.-T. Lau, D. De Rossi, G. Wallace, D. Diamond, Smart Nanotextiles: A Review of Materials and Applications, MRS. Bull. 32 (2007) 434. [6] K. Jost, C.R. Perez, J.K. McDonough, V. Presser, M. Heon, G. Dion, Y. Gogotsi, Carbon coated textiles for flexible energy storage, Energy Environ. Sci. 4 (2011) 5060.

[7] M. Winter, R.J. Brodd, What are batteries, fuel cells, and supercapacitors?, Chem. Rev. 104 (2004) 4245.

[8] J. Zhong, Y. Zhang, Q. Zhong, Q. Hu, B. Hu, Z.L. Wang, J. Zhou, Fiber-based generator for wearable electronics and mobile medication, ACS Nano 8 (2014) 6273. [9] S.J. Kim, J.H. We, B.J. Cho, A wearable thermoelectric generator fabricated on a glass fabric, Energy Environ. Sci. 7 (2014) 1959.

[10] W. Zeng, L. Shu, Q. Li, S. Chen, F. Wang, X.-M. Tao, Fiber-based wearable electronics: A review of materials, fabrication, devices, and applications, Adv. Mater. 26 (2014) 5310.

[11] K. Jost, G. Dion, Y. Gogotsi, Textile energy storage in perspective, J. Mater. Chem. A 2 (2014) 10776.

[12] L. Hu, M. Pasta, F.L. Mantia, L. Cui, S. Jeong, H.D. Deshazer, J.W. Choi, S.M. Han, Y. Cui, Stretchable, porous, and conductive energy textiles, Nano Lett. 10 (2010) 708. 
[13] G. Yu, L. Hu, M. Vosgueritchian, H. Wang, X. Xie, J.R. McDonough, X. Cui, Y.

Cui, Z. Bao, Solution-processed graphene/ $\mathrm{MnO}_{2}$ nanostructured textiles for high-performance electrochemical capacitors, Nano Lett. 11 (2011) 2905.

[14] K. Wang, P. Zhao, X.M. Zhou, H.P. Wu, Z.X. Wei, Flexible supercapacitors based on cloth-supported electrodes of conducting polymer nanowire array/SWCNT composites, J. Mater. Chem. 21 (2011) 16373.

[15] W.W. Liu, X.B. Yan, J.W. Lang, C. Peng, Q.J. Xue, Flexible and conductive nanocomposite electrode based on graphene sheets and cotton cloth for supercapacitor, J. Mater. Chem. 22 (2012) 17245.

[16] K. Jost, D. Stenger, C.R. Perez, J.K. McDonough, K. Lian, Y. Gogotsi, G. Dion, Knitted and screen printed carbon-fiber supercapacitors for applications in wearable electronics, Energy Environ. Sci. 6 (2013) 2698.

[17] B. Yue, C. Wang, X. Ding, G.G. Wallace, Polypyrrole coated nylon lycra fabric as stretchable electrode for supercapacitor applications, Electrochim. Acta 68 (2012) 18.

[18] B. Yue, C. Wang, X. Ding, G.G. Wallace, Electrochemically synthesized stretchable polypyrrole/fabric electrodes for supercapacitor, Electrochim. Acta 113 (2013) 17.

[19] L.G. Zhu, W.L. Xu, L. Wu, Y.Y. Sun, M.X. Li, J. Xu, Z.K. Bai, G.J. Liang, L. Liu, D. Fang, Cotton fabrics coated with lignosulfonate-doped polypyrrole for flexible supercapacitor electrodes, RSC Adv. 4 (2014) 6261.

[20] Y.S. Wang, S.M. Li, S.T. Hsiao, W.H. Liao, P.H. Chen, S.Y. Yang, H.W. Tien, C.C.M. Ma, C.C. Hu, Integration of tailored reduced graphene oxide nanosheets and electrospun polyamide-66 nanofabrics for a flexible supercapacitor with high-volumeand high-area-specific capacitance, Carbon 73 (2014) 87.

[21] Q. Niu, K. Gao, Z. Shao, Cellulose nanofiber/single-walled carbon nanotube hybrid non-woven macrofiber mats as novel wearable supercapacitors with excellent stability, tailorability and reliability, Nanoscale 6 (2014) 4083.

[22] H. Cheng, Z. Dong, C. Hu, Y. Zhao, Y. Hu, L. Qu, N. Chen, L. Dai, Textile electrodes woven by carbon nanotube-graphene hybrid fibers for flexible 
electrochemical capacitors, Nanoscale 5 (2013) 3428.

[23] J. Bae, M.K. Song, Y.J. Park, J.M. Kim, M. Liu, Z.L. Wang, Fiber supercapacitors made of nanowire-fiber hybrid structures for wearable/flexible energy storage, Angew. Chem. Int. Ed. 50 (2011) 1683.

[24] Y. Fu, X. Cai, H. Wu, Z. Lv, S. Hou, M. Peng, X. Yu, D. Zou, Fiber supercapacitors utilizing pen ink for flexible/wearable energy storage, Adv. Mater. 24 (2012) 5713.

[25] V.T. Le, Y.H. Lee, H. Kim, A. Ghosh, J. Kim, J. Chang, Q.A. Vu, D.T. Pham, J.-H. Lee, S.-W. Kim, Coaxial fiber supercapacitor using all-carbon material electrodes, ACS Nano 7 (2013) 5940.

[26] J.A. Lee, R.H. Baughman, S.J. Kim, M.K. Shin, S.H. Kim, H.U. Cho, G.M. Spinks, G.G. Wallace, M.D. Lima, X. Lepró, M.E. Kozlov, Ultrafast charge and discharge biscrolled yarn supercapacitors for textiles and microdevices, Nat. Commun. 4 (2013) 1970.

[27] Y. Meng, Y. Zhao, C. Hu, H. Cheng, Y. Hu, Z. Zhang, G. Shi, L. Qu, All-graphene core-sheath microfibers for all-solid-state, stretchable fibriform supercapacitors and wearable electronic textiles, Adv. Mater. 25 (2013) 2326. [28] Z. Yang, J. Deng, X. Chen, J. Ren, H. Peng, A highly stretchable, fiber-shaped supercapacitor, Angew. Chem. Int. Ed. 52 (2013) 13453.

[29] S. Biswas, L.T. Drzal, Multilayered Nanoarchitecture of Graphene Nanosheets and Polypyrrole Nanowires for High Performance Supercapacitor Electrodes, Chem. Mater. 22 (2010) 5667.

[30] C. Xu, J. Sun, L. Gao, Synthesis of novel hierarchical graphene/polypyrrole nanosheet composites and their superior electrochemical performance, J. Mater. Chem. $21(2011) 11253$.

[31] W.S. Hummers Jr, R.E. Offeman, Preparation of graphitic oxide, J. Am. Chem. Soc. 80 (1958) 1339.

[32] S. Park, J.H. An, R.D. Piner, I. Jung, D.X. Yang, A. Velamakanni, S.T. Nguyen, R.S. Ruoff, Aqueous suspension and characterization of chemically modified graphene sheets, Chem. Mater. 20 (2008) 6592. 
[33] P. Petersen, R. Helmer, M. Pate, J. Eichhoff, Electronic textile resistor design and fabric resistivity characterization, Text. Res. J. 81 (2011) 1395.

[34] Y. Xu, Z. Lin, X. Huang, Y. Liu, Y. Huang, X. Duan, Flexible solid-state supercapacitors based on three-dimensional graphene hydrogel films, ACS Nano 7 (2013) 4042.

[35] S. Pei, H.-M. Cheng, The reduction of graphene oxide, Carbon 50 (2012) 3210.

[36] S. Stankovich, D.A. Dikin, R.D. Piner, K.A. Kohlhaas, A. Kleinhammes, Y. Jia, Y. Wu, S.T. Nguyen, R.S. Ruoff, Synthesis of graphene-based nanosheets via chemical reduction of exfoliated graphite oxide, Carbon 45 (2007) 1558.

[37] K.N. Kudin, B. Ozbas, H.C. Schniepp, R.K. Prud'homme, I.A. Aksay, R. Car, Raman spectra of graphite oxide and functionalized graphene sheets, Nano Lett. 8 (2008) 36.

[38] L. Buglione, E.L.K. Chng, A. Ambrosi, Z. Sofer, M. Pumera, Graphene materials preparation methods have dramatic influence upon their capacitance, Electrochem. Commun. 14 (2012) 5.

[39] J. Zhang, H. Yang, G. Shen, P. Cheng, J. Zhang, S. Guo, Reduction of graphene oxide via L-ascorbic acid, Chem. Commun. 46 (2010) 1112.

[40] M.J. Fernández-Merino, L. Guardia, J.I. Paredes, S. Villar-Rodil, P. Solís-Fernández, A. Martínez-Alonso, J.M.D. Tascón, Vitamin C is an ideal substitute for hydrazine in the reduction of graphene oxide suspensions, J. Phys. Chem. C 114 (2010) 6426.

[41] T. Kerr, K. Duncan, L. Myers, Post fire materials identification by micro-Raman spectroscopy and principal components analysis, J. Anal. Appl. Pyrol. 102 (2013) 103. [42] Y.-R. Kang, Y.-L. Li, F. Hou, Y.-Y. Wen, D. Su, Fabrication of electric papers of graphene nanosheet shelled cellulose fibres by dispersion and infiltration as flexible electrodes for energy storage, Nanoscale 4 (2012) 3248.

[43] K. Xie, B. Wei, Materials and structures for stretchable energy storage and conversion devices, Adv. Mater. 26 (2014) 3592.

[44] M. Pasta, F. La Mantia, L. Hu, H.D. Deshazer, Y. Cui, Aqueous supercapacitors on conductive cotton, Nano Res. 3 (2010) 452. 
[45] Y.-C. Liu, B.-J. Hwang, W.-J. Jian, R. Santhanam, In situ cyclic voltammetry-surface-enhanced Raman spectroscopy: studies on the doping-undoping of polypyrrole film, Thin Solid Films 374 (2000) 85.

[46] Y.-J. Peng, T.-H. Wu, C.-T. Hsu, S.-M. Li, M.-G. Chen, C.-C. Hu, Electrochemical characteristics of the reduced graphene oxide/carbon nanotube/polypyrrole composites for aqueous asymmetric supercapacitors, J. Power Sources 272 (2014) 970.

[47] C. Zhao, C. Wang, Z. Yue, K. Shu, G.G. Wallace, Intrinsically stretchable supercapacitors composed of polypyrrole electrodes and highly stretchable gel electrolyte, ACS Appl. Mater. Interfaces 5 (2013) 9008.

[48] C. Zhao, C. Wang, R. Gorkin, S. Beirne, K. Shu, G.G. Wallace, Three dimensional (3D) printed electrodes for interdigitated supercapacitors, Electrochem. Commun. 41 (2014) 20.

[49] L.Q. Fan, G.J. Liu, J.H. Wu, L. Liu, J.M. Lin, Y.L. Wei, Asymmetric supercapacitor based on graphene oxide/polypyrrole composite and activated carbon electrodes, Electrochim. Acta 137 (2014) 26. 